\title{
LA CIENCIA COMO ACCIÓN
}

\author{
Juan Carlos Moreno Ortiz \\ doi:10.11144/Javeriana.uph31-63.lcca
}

El títUlo DE ESTA LECCIÓN SUGIERE entender el quehacer científico como una forma de acción, específicamente, la que se coloca en juego en el "hacer". Buena parte de los filósofos que estudiamos, especialmente los modernos, nos han cimentado la idea de que la ciencia es producto de una razón desvinculada de la acción, de que el entendimiento moldea el conocimiento científico y de que la acción, en su sentido productivo, poco tiene que ver con el desarrollo de tal conocimiento. Yo quiero plantear lo contrario a partir del camino que desde el siglo XVII mostró Giambattista Vico, a través de su expresión: Verum ipsum factum, es decir, el conocimiento verdadero es el que hacemos, el que producimos de forma activa. Esto quiere decir que el conocimiento verdadero no se reduce a una actividad pura de nuestro pensamiento, sino que es algo activo, operativo, material, un hacer productivo.

Cuando se habla de la "acción" en la ciencia, se abre el abanico de orientaciones hacia varias opciones. En primer lugar se puede pensar, desde un punto de vista muy común, que propone una reflexión sobre la forma como se aplican los conocimientos científicos o sobre las implicaciones prácticas que tendría la ciencia. Desde una perspectiva más filosófica, muchos podrían suponer que el problema de la acción en la ciencia tendría que ver especialmente con el sentido ético, axiológico o político implicado en el desarrollo del conocimiento científico, planteado por autores como Nicolás Rescher, Evandro Agazzi o Javier Echeverría, por mencionar algunos. Desde un punto de vista más analítico, se puede pensar en la extensión de una teoría general de la acción al campo concreto del conocimiento científico; es decir,

\footnotetext{
${ }^{*}$ Pontificia Universidad Javeriana, Bogotá, Colombia.

Para citar este artículo: Moreno Ortiz, J.C (2014). La ciencia como acción. Universitas Philosophica, 31(63), pp. 289-301, ISSN 0120-5323, ISSN en línea 2346-2426, doi: 10.11144/Javeriana.uph31-63.lcca
} 
en el planteamiento de un análisis sobre el carácter causal o no causal de la acción, sobre su sentido intencional, sobre los agentes, sobre la justificación racional de la acción o, también, sobre el carácter normativo involucrado en ella. Por este camino se tendría que acudir a autores como Davidson o von Wright.

Pero también, de una forma muy general, se habla de acción en la ciencia cuando se quiere, a manera de contrapeso, mostrar lo que significa estudiarla desde el punto de vista de sus prácticas, de sus procesos de producción, de la historia, de la sociología u otra disciplina que devele sus prácticas, y no entendida como un producto, ni como un conjunto de teorías o resultados finales. En este sentido se puede ubicar el título del libro de Bruno Latour (1987), La ciencia EN acción, como un tipo de reflexión que coloca la atención en la forma como ha sido practicada la ciencia, a partir del giro acontecido desde Thomas Kuhn hacia el estudio de las prácticas científicas.

También, desde un punto de vista muy específico, se podría orientar la reflexión sobre la acción en la ciencia por el camino que plantea Alfredo Marcos con su texto Ciencia y acción (2010). Este autor propone comprender la racionalidad científica, a partir de Aristóteles, no como teórica sino como práctica prudencial. Es decir, como un tipo de racionalidad falible, de acuerdo con lo que han mostrado Peirce y Popper, que puede satisfacer condiciones epistémicas especiales procediendo de manera prudencial.

Por ninguno de estos caminos se va a orientar la presente reflexión. Sin desconocer estas tendencias, esta disertación pretende comprender el conocimiento científico como acción en su sentido más material y mundano, es decir, como "hacer productivo". Recalco la fuerza de las palabras "como acción" propuestas en el título de esta lección, para indicar que mi intención es hacer un planteamiento sobre una forma de entender el conocimiento científico y no una reflexión sobre la relación de la ciencia con el ámbito de la acción.

A manera de tesis quiero plantear que el quehacer científico en el sentido de un hacer productivo tiene una función epistemológica fundamental: quehacer y conocer en la ciencia no son dos instancias separadas, sino íntimamente vinculadas. Pero, sobre todo, quiero analizar cómo el tipo de acción característica del hacer productivo cumple un papel mediador en la producción de conocimiento. 
Es lamentable que nuestra tradición filosófica occidental se haya parcializado hacia una concepción mentalista, racionalista $\mathrm{y}$ representacionalista del conocimiento, que haya soslayado el papel del hacer productivo como un tipo de actividad estéril y secundaria para el desarrollo del conocimiento, invisibilizando una buena parte de lo que se encuentra involucrado en el quehacer intelectual, tanto en la ciencia como en muchos otros tipos de conocimiento. Esta tradición ha sostenido una distinción radical entre racionalidad teórica y racionalidad práctica, haciéndonos suponer que la comprensión del conocimiento científico solo pertenece al ámbito de la racionalidad teórica, lo que afianza la vieja separación entre ciencia y técnica.

Sin embargo, procesos activos como el hacer experimental, el hacer instrumental, el hacer técnico y tecnológico, los procesos de codificación y registro de datos, etc., no son instancias triviales en términos epistemológicos, subordinadas al desarrollo teórico, sino procesos activos que pueden cumplir un papel cognitivo importante en el desarrollo de la ciencia. Y con mayor razón lo son también los demás procesos activos habituales del quehacer intelectual y científico, es decir, el hacer cálculos, inferencias, descubrimientos, justificaciones, debates, etc.

El papel epistemológico del hacer y el papel activo del conocer se contraponen a las suposiciones ampliamente asumidas por la marcada orientación racionalista asumida en la Filosofía de la Ciencia, desde el positivismo lógico hasta Kuhn, pero tienen un largo antecedente filosófico y científico, desde los siete sabios griegos, pasando por autores como Filón, Ficino, Da Vinci, Cardano, Bacon, Galileo, como lo muestra Rodolfo Mondolfo en su texto Verum Factum (1971), hasta autores más recientes como Piaget, y actuales como Ian Hacking, Bruno Latour, Peter Galison, Andrew Pickering. Para comprender el sentido de mi planteamiento propongo realizar enseguida una rápida revisión del tipo de función epistemológica analizada en la ciencia como hacer, por algunos importantes y conocidos autores de la tradición filosófica.

Rodolfo Mondolfo en el texto mencionado explora los antecedentes de la concepción activista del conocimiento científico, desde los siete sabios griegos hasta Marx, condensada en la expresión Verum Factum de Giambattista Vico. Seguramente Vico ha sido el autor clásico que mejor ha analizado el sentido epistemológico del hacer. Su famosa expresión, entendida como: solo conocemos verdaderamente lo que hacemos, es una 
clara expresión de su epistemología activista. Para el filósofo napolitano el factum no es una realidad independiente de nosotros, que se presenta a nuestra observación, sino algo que nosotros mismos hacemos, por lo que podemos conocerla verdaderamente.

Según Mondolfo (1971, p. 21), para Vico, la expresión "factum no significa una realidad (de acontecimientos o de cosas) que se ofrece al sujeto cognoscente, sino que quiere señalar la acción creadora del sujeto, que conquista verdaderamente el conocimiento (verum) en cuanto lo hace, es decir, en cuanto él mismo es el autor y como tal conoce el producto de su propia obra". Factum es la condición esencial para la conquista del verdadero conocimiento (verum).

Ahora bien, la argumentación de Mondolfo continúa con las palabras de Vico en la segunda respuesta de su texto Discursos inaugurales: "por lo tanto, el criterio de tener ciencia de una cosa es llevarla a efecto... El conocimiento del [hecho] y la operación es una misma cosa" (Vico citado por Mondolfo, 1971, p. 22). Con esto, Vico no está diciendo que el conocer de forma verdadera precede al hacer, o que el hacer precede el conocer, sino que factum y verum, hacer y conocer verdaderamente, son equivalentes, por ello, "lo verdadero es una misma cosa con lo hecho [y] en tanto Dios es lo primero verdadero en cuanto es el primer hacedor o creador" (Vico citado por Mondolfo, 1971, p. 22).

Este doble sentido del factum no solo como algo dado, sino como algo que se hace y se produce, lo señala Latour en su famoso texto La vida en el laboratorio, del siguiente modo:

«Hecho» puede tener dos significados contradictorios [...] «hecho» se deriva de la raíz facere, factum (hacer o fabricar). Por otro lado, se considera que «hecho» se refiere a alguna entidad objetivamente independiente que, en virtud de su «carácter externo» no se puede modificar a voluntad y no es susceptible de cambio bajo cualesquiera circunstancias. (Latour, 1995, p. 196)

Un tema de gran relevancia en varios estudios históricos recientes ha sido el análisis de la constitución de lo que se llama "hechos" en la ciencia. Específicamente, me refiero a la famosa investigación de Shapin y Shaffer sobre la bomba de vacío (2005), y a la investigación ya mencionada de Latour (1995). En ambos estudios se analiza el surgimiento o el nacimiento 
de objetos de estudio que han gozado posteriormente del estatuto de hechos, como el vacío o la hormona liberadora de la tirotropina. Su constitución de hechos se encuentra ligada al establecimiento de la evidencia a través de complejos procesos instrumentales y experimentales.

Volviendo a nuestra revisión sobre los antecedentes del tema, el carácter epistémico del hacer hunde sus raíces hasta la antigüedad griega, como lo señala Mondolfo. Este muestra que durante el siglo $\mathrm{V}$ y la edad de la cultura sofística, el significado de techné equivale al de episteme y que, incluso en Platón, se evidencia una promiscuidad terminológica entre esos dos conceptos (Mondolfo, 1971, p. 14).

Según Mondolfo (1971, p. 14):

Un documento sumamente significativo, tal vez el más significativo de todos, sobre el valor epistemológico que tiene el recurso a las técnicas en el antiguo naturalismo griego, lo constituye el escrito hipocrático, De los alimentos. El escritor hipocrático al presentar las técnicas del forjador, del batanero, el remendón, del carpintero, del constructor, del músico, del curtidor, del cestero, del refinador de oro, del escultor, del alfarero, del copista, como imitaciones de los procesos naturales del organismo humano (...) convierte en teoría consciente y explícita la utilización que hacían todos los presocráticos de las sugerencias de la técnica para la interpretación de la naturaleza.

Para Hipócrates las técnicas imitan los procesos naturales del organismo humano y por eso nos revelan sus secretos. La técnica interpreta la naturaleza. Entonces, existe una semejanza entre los procesos fisiológicos y las técnicas que proviene de una misteriosa enseñanza divina sobre la que no tienen conciencia los hombres: "los hombres no comprenden cómo se puede observar lo oculto mediante lo manifiesto" (Hipócrates citado por Mondolfo, 1971, p. 15).

Lo oculto y lo desconocido son los procesos de la naturaleza que se desarrollan sin la intervención alguna del hombre; lo manifiesto y lo conocido, procesos de la técnica, que los hombres mismos realizan y que, por consiguiente, conocen verdadera y precisamente porque los realizan, los hacen. Para Hipócrates, pues, el hacer penetra en la naturaleza de las cosas, mientras la contemplación pasiva no logra un conocimiento de la naturaleza. Los hombres "conocen lo que hacen e ignoran lo que imitan" 
(Hipócrates citado por Mondolfo, 1971, p. 15). Por ello, los hombres no saben que pueden observar lo invisible por medio de lo visible, puesto que al emplear técnicas semejantes a la naturaleza humana no se dan cuenta de ello. La mente de los dioses, en efecto, les enseñó a imitar los procesos de la naturaleza, conociendo lo que hacen, pero sin conocer lo que imitan.

La exploración en los autores antiguos que le asignan un sentido epistemológico al hacer podría ser muy amplia y, por lo mismo, no la vamos a realizar en esta lección. También se encuentran antecedentes de este planteamiento en algunos autores modernos como Da vinci, Cardano, Galileo, Bacon, Campanella. Como nos lo recuerda Hacking, Bacon enseñaba que, "no sólo deberíamos observar la naturaleza en vivo, sino que también deberíamos "torcerle la cola al león", esto es, manipular nuestro mundo para aprender sus secretos" (Hacking, 1996, p. 177).

Algo similar se puede ver en Galileo. Sus experimentos con los planos inclinados fueron importantes no porque permitieran contrastar empíricamente una hipótesis previamente formulada sobre el movimiento uniformemente acelerado, como se esperaría desde una epistemología kantiana; tampoco lo son porque develaran a la observación algún tipo de relación entre el tiempo y el espacio no advertida por otro camino. Fueron importantes porque mediaron la comprensión de situaciones físicas difíciles de enfrentar por el simple razonamiento, como el carácter infinitesimal y continuo del movimiento. Las múltiples manipulaciones experimentales realizadas por Galileo permitieron de forma activa u operativa encontrar las soluciones físicas y matemáticas del movimiento, que la mera razón no podía hallar mientras permaneciera hundida en las paradojas que se desprenden del análisis racional del carácter infinitesimal y continuo del movimiento, paradojas que desde muy antiguo había advertido ya Zenón de Elea ${ }^{1}$. También, para Galileo, el telescopio fue más que un instrumento,

\footnotetext{
${ }^{1}$ A esta conclusión se puede llegar a partir del análisis de las cuatro dificultades que Galileo enfrentó antes de considerar aceptada la ley de la caída de los cuerpos en su texto de los Discorsi (Galileo, 1988a). Estas dificultades tienen que ver con una comprensión especialmente lógica y filosófica del movimiento de caída de los cuerpos. Para la gran mayoría de los especialistas sobre el tema ha quedado claro que Galileo no dedujo racionalmente esa ley, ni la infirió o justificó a través de los experimentos con el plano inclinado, a pesar de haber realizado cuidadosamente muchos experimentos de ese tipo, como consta en los análisis de sus manuscritos (Galileo, 1988b). Lo más seguro es que los experimentos cumplieron fundamentalmente una función heurística.
} 
pues cambió la manera de establecer la experiencia en la astronomía. Fue un mediador, un "sidereus nuncius", o un mensajero celeste entre dos mundos que antes se encontraban totalmente separados: el mundo lunar del orden celeste y el mundo sublunar de la experiencia humana.

Siguiendo con el análisis del surgimiento de la experimentación en el siglo XVII, el barómetro de Torricelli y la bomba de vacío construida por Boyle y Hooke fueron importantes porque instauraron un nuevo espacio para definir y analizar el vacío, no ya como un concepto metafísico, tal como se analizaba en la física aristotélica, sino como un concepto físico, un efecto que se puede producir. La elaboración del efecto supuso el establecimiento de condiciones empíricas para el desarrollo de un nuevo campo de la física como la neumática, y de una nueva forma de análisis de la idea de vacío importante en la nueva física planteada por Newton (Shapin y Shaffer, 2005).

En estos casos distintos la elaboración de experimentos y de instrumentos sirvió, utilizando un término arquimédico, de palanca cognitiva. “¡Eureka!”, o el proceso de descubrimiento/invención/producción, no fueron en estos casos meros saltos creativos del entendimiento, sino saltos creativos mediados por el hacer productivo. En ellos el hacer cumplió un papel mediador para el acto poiético. Por lo que dicho acto no fue solo un descubrimiento sino también una producción material.

En relación con el análisis del papel epistemológico del hacer no podemos dejar de mencionar a un autor contemporáneo como Piaget. Desde la perspectiva de una teoría del desarrollo, Piaget ha mostrado cómo algunas nociones matemáticas como la adición, la sustracción, la conmutación, la transitividad, etc. se desarrollan en el niño a través de acciones relacionadas con la manipulación de objetos en acciones como la utilización de los dedos de las manos para contar, o la reunión y separación, la distribución y clasificación de objetos. En las operaciones lógico-matemáticas se procede de la aplicación de operaciones a objetos físicos, a la interiorización de operaciones manipulables simbólicamente. De las acciones físicas con objetos, a las acciones mentales con símbolos.

En términos generales, Piaget, en su artículo "El mito del origen sensorial de los conocimientos científicos", afirma lo siguiente (1971, p. 88):

Nuestros conocimientos no provienen únicamente ni de la sensación ni de la percepción, sino de la totalidad de la acción con respecto de la cual la 
percepción solo constituye la función de señalización. En efecto, lo propio de la inteligencia no es contemplar, sino transformar y su mecanismo es esencialmente operatorio.

Incluso, el desarrollo de las nociones matemáticas no solo se moldea a través de las acciones, sino que tiene como condición una degradación inicial de lo representacional, en lugar de un mayor desarrollo. La acción requiere de percepciones, pero únicamente de las que Piaget denomina propioceptivas, que cumplen una función especialmente relacionada con la señalización de la acción.

Lo importante no son las acciones consideradas aisladamente, sino el esquema de dichas acciones. Sin embargo, el esquema no es, como para buena parte de la epistemología moderna, producto de las representaciones perceptuales, sino de la acción misma. "El esquema no sale de la percepción, sea propioceptiva o de otro tipo; el esquema es el resultado directo de la generalización de las acciones mismas y no de su percepción; como tal, el esquema no es perceptible en absoluto" (Piaget, 1971, p. 91).

Piaget plantea que el esquema es más rico que la percepción correspondiente. Por ejemplo, la noción de espacio es más rica que la percepción del espacio, y esto por dos razones: de un lado, porque el esquema o la noción no consiste simplemente en traducir el dato perceptivo, sino también en corregirlo; de otro, por todo lo que la acción añade a la percepción.

Así pues, de los resultados precedentes podemos sacar dos conclusiones. Por una parte, los conocimientos no proceden nunca exclusivamente de la sensación o de la percepción, sino también de los esquemas de acciones o de los esquemas operatorios, que son, tanto unos como otros, irreductibles a la percepción sin más. Por otra parte, la percepción misma no consiste en una simple lectura de los datos sensoriales, sino que implica una organización activa en la que intervienen decisiones y pre-inferencias y que se debe a la influencia sobre la percepción como tal del esquematismo de las acciones o de las operaciones. (...)

(...) en cada una de sus manifestaciones el conocimiento científico refleja la inteligencia humana que, por su naturaleza operatoria, procede de la acción completa. (Piaget, 1971, pp. 111-112)

Con esta rápida revisión de autores hemos mostrado que la idea de identificar un sentido epistemológico en el hacer tiene un largo antecedente. 
No obstante, para comprender bien cómo el hacer es un camino para el conocimiento en la ciencia es preciso entender cómo en el hacer se produce una mediación o un proceso operatorio que contribuye a la constitución de la experiencia.

Estaidea se comprendemejor si hacemos un contraste con la epistemología kantiana. Para Kant la sensibilidad es pasiva, y el entendimiento, activo. De acuerdo con lo que él denomina "la espontaneidad del entendimiento" solo este puede producir conceptos fuera de la experiencia o no derivados de ella. De este modo, la sensibilidad, mediada por el hacer productivo, no tendría un sentido pasivo sino activo. En el terreno del hacer la espontaneidad, la condición de elaborar conceptos y establecer la experiencia, se extendería más allá del papel exclusivo del entendimiento, para ubicarse en un campo de mediación determinado a través de la interacción entre lo que la sensibilidad dispone, lo que el entendimiento produce y lo que el hacer media.

La complejidad de las ideas acerca del modocomo se producelamediación del hacer y de cómo este asume un sentido positivo epistemológico, se puede comprender en el ámbito específico del trabajo experimental e instrumental, como campos privilegiados del hacer en la ciencia. Ian Hacking y Andrew Pickering nos ayudan en esta tarea.

El desarrollo de la ciencias experimentales, para Hacking y Pickering, no es el despliegue de un proceso teórico que se hace práctico a través de los procesos experimentales e instrumentales, sino que es el ajuste mutuo de elementos teórico-prácticos como las teorías, el desarrollo de aparatos, el establecimiento de registros, etc. Este ajuste no se produce a priori, de antemano, sino en el curso de las acciones.

Hacking anota que el trabajo científico en las ciencias de laboratorio guarda correspondencia con los ajustes "robustos" de diferentes elementos heterogéneos obtenidos en las intervenciones, en el tiempo real de las prácticas, y no con la adecuación a algo predefinido o preestablecido. Al respecto el filósofo sostiene que:

El trabajo de laboratorio requiere que consigamos un ajuste robusto entre aparatos, creencias y análisis de datos y teorías. Antes de que se haya alcanzado un ajuste robusto, no está determinado cuál será ese ajuste. Ni determinado por cómo es el mundo, ni determinado por la tecnología 
ahora existente, ni determinado por las prácticas sociales de los científicos, ni determinado por intereses o redes, ni determinado por el genio, ni determinado por nada (...).

No se trata de elegir una teoría, o un instrumental o una fenomenología entre las disponibles, “(...) sino de trastear con la teoría, los aparatos y las descripciones de lo que están haciendo los aparatos”. (Hacking, 2001, pp. 125-126)

De manera detallada Pickering (1995) nos explica el proceso mediador del hacer como un proceso performativo, que denomina "el modelamiento de la práctica". Más allá del ajuste entre el conocimiento subjetivo y el mundo objetivo, o entre las teorías y los hechos observables, se trata de un proceso de acomodaciones para resolver las resistencias y buscar ajustes robustos, practicado a la manera de una "puesta a punto", en sentido análogo de la 'sintonización' (tuning) de una emisora en la radio o de la 'sincronización' de un automóvil. Esas adaptaciones o ajustes no se pueden definir de antemano, sino en la práctica o en el curso de las acciones.

Los contornos de la agencia material nunca se conocen de antemano de manera decisiva, los científicos tienen que explorarlos en su trabajo continuamente, los problemas surgen siempre y tienen que ser resueltos en el desarrollo, por ejemplo, de nuevas máquinas. Y tales soluciones, -si se encuentran-toman la forma, como mínimo, de un tipo de material delicado posicionándose o poniéndose a punto (tuning), donde utilizo "poniéndose a punto" (tuning) en el sentido de sintonización de un radio o del motor del coche, con la salvedad de que el carácter de la "señal" no se conoce de antemano en la investigación científica. (Pickering, 1995, p. 14)²

Lo más importante de esta concepción es que el carácter de la señal o, dicho de otro modo, de la especificación de los contenidos y de las trayectorias materiales, no se conoce de antemano o no es producto de la red conceptual previa. Esos contenidos y trayectorias materiales solo se especifican en la interacción entre artefactos, ideas y marcas, para producir el fenómeno, pues no son la expresión de un ajuste prediseñado.

\footnotetext{
2 "The contours of material agency are never decisively known in advance, scientists continually have to explore them in their work, problems always arise and have to be solved in the development of, say, new machines. And such solutions -if they are found at all-take the form, at minimum, of a kind of delicate material positioning or tuning, where I use "tuning" in the sense of tuning a radio set or car engine, with the caveat that the character of the "signal" is not known in advance in scientific research".
} 
Estos mutuos ajustes se dan en el tiempo real de la práctica, es decir, no sobrepasan el transcurso de las acciones. Por ejemplo, un automóvil solo se puede sincronizar ensuciándose las manos o en la acción directa de modificar las partes del automóvil guiado por la habilidad práctica del mecánico. Así, las expresiones particulares de la máquina se acoplan con la experiencia y las habilidades adquiridas por el agente a través del entrenamiento. Con todo, desde este punto de vista, la ciencia no es un mero pensar y la tecnología no se reduce a un hacer subordinado, guiado por el pensar.

No quiero extender esta reflexión más de lo necesario y justo para la ocasión. El interés primordial era suscitar el debate sobre algunas ideas y no analizar de manera extensa un tema. Entonces, quiero finalizar mi intervención haciendo una sencilla exhortación, desde los planteamientos que he expuesto, para el desarrollo del ejercicio filosófico. Específicamente deseo llamar la atención sobre el carácter activo del quehacer filosófico. A los filósofos nos han estigmatizado como pensadores puros. La imagen del filósofo reflexivo, como la de la escultura del pensador de Rodin, manifiesta solo un aspecto de la forma de conocer de esta disciplina, y omite otra forma importante que queda consignada en la frase ordinaria: "hacer filosofía". En efecto, ni la filosofía ni la ciencia tienen su asidero en un "pensar" separado de la acción como "hacer" productivo. Ellas son un "hacer" que produce ideas, teorías, modelos interpretativos, etc.

Tanto la ciencia como la filosofía se "hacen", en todo el sentido de la palabra, en las disertaciones emprendidas con los amigos y colegas mientras se toma un café; en las interacciones sostenidas en el aula de clase; en el intercambio de ideas realizado en un congreso, simposio o foro; en la paciente y dispendiosa labor de enfrentarse a una pantalla en blanco para escribir un artículo o una ponencia; en el tortuoso trabajo de hacer o dirigir una tesis; en el ingrato esfuerzo de conseguir recursos para la investigación con Colciencias. Esto es, en labores que despliegan muchas formas del hacer productivo.

Espero que esta sencilla Lectio haya permitido entender el significado epistemológico que tienen las palabras "hacer" y "producir", en los dominios de la ciencia y la filosofía, y que motiven la curiosidad filosófica de algunos, la comprensión más amplia del quehacer científico y filosófico y, sobre todo, el "hacer filosofía" en su sentido activo, operativo y productivo justo en el momento en que inauguramos un nuevo semestre académico. 


\section{Referencias}

Galileo, G. (1988a). Discursos y demostraciones matemáticas en torno a dos nuevas ciencias relativas a la Mecánica y los Movimientos Locales. C. Azcárate, D. García \& J. Romo (Eds.), Galileo Galilei: La nueva ciencia del movimiento. Selección y edición crítica de los Discorsi, Vol. 2. Barcelona: Edición de la Universidad Politécnica de Cataluña y Publicación de la Universidad Autónoma de Barcelona.

Galileo, G. (1988b). Manuscritos. C. Azcárate, D. García \& J. Romo (Eds.), Galileo Galilei: La nueva ciencia del movimiento. Selección y edición crítica de los Discorsi (pp. 147-153/159-169/363-448). Barcelona: Edición de la Universidad Politécnica de Cataluña y Publicación de la Universidad Autónoma de Barcelona.

Hacking, I. (2001). ¿La Construcción Social de Qué? (Trad. J. Sánchez). Barcelona/Buenos Aires: México: Paidós.

HAcking, I. (1999). The Social Construction of WHAT? Cambridge/London: Harvard University Press.

HaCkING, I. (1996). Representar e Intervenir. (Trad. S. Martínez). México: Paidós.

Kunn, T.S. (1997). La estructura de las revoluciones cientificas. (Trad. A. Contín). Madrid: F.C.E.

Latour, B. \& Woolgar, S. (1995). La vida en el laboratorio. La construcción de los hechos cientificos. (Trad. S. Pérez). Madrid: Alianza.

Latour, B. \& Woolgar, S. (1986). Laboratory Life: The Construction of Scientific Facts. Princeton: Princeton University Press.

Latour, B. (1992). Ciencia en acción: cómo seguir a los cientificos e ingenieros a través de la sociedad. Barcelona: Labor.

Latour, B. (1987). Science in Action: How to Follow Scientists and Engineers through Society. Cambridge: Harvard University Press.

Lynch, M. (1993). Scientific Practice and Ordinary Action: Ethnomethodology and Social Studies of Science. New York: Cambridge University Press.

Marcos, A. (2010). Ciencia y acción. Una filosofía práctica de la ciencia. México: F.C.E.

Mondolfo, R. (1971). Verum Factum, desde antes de Vico hasta Marx. México: Siglo XXI. 
Piaget, J. (1971). El mito del origen sensorial de los conocimientos científicos. Psicología y epistemología. Barcelona: Ariel.

Pickering, A. (1995). The Mangle of Practice. Time, Agency, and Science. Chicago: The University of Chicago Press.

Pickering, A. (Ed.). (1992). Science as Practice and Culture. Chicago/London: University of Chicago Press.

Pickering, A. (1989). Living in the Material World. D. Gooding, P. Trevor \& S. Schaffer (Eds.). The Uses of Experiment. New York: Cambridge University Press.

Schaffer, S. \& Shapin, S. (2005). El Leviathan y la bomba de vacio Hobbes, Boyle y la vida experimental. (Trad. A. Buch). Quilmes: Universidad Nacional de Quilmes Editorial.

Schatzki, T. et al. (2001). The Practice Turn in Contemporary Theory. London/ New York: Routledge.

Vico, G. (2008). Ciencia Nueva. Madrid: Tecnos.

VIco, G. (2002). Obras, Vols. 1 y 2. Barcelona: Ánthropos. 